\title{
OPEN Abundant and diverse Tetrahymena species living in the bladder traps of aquatic carnivorous Utricularia \\ plants
}

Received: 23 May 2019

Accepted: 6 September 2019

Published online: 20 September 2019

\author{
Chao-Yin Cheng ${ }^{1}$, Shang-Lin Chang ${ }^{2}$, I-Ting Lin ${ }^{1}$ \& Meng-Chao Yao ${ }^{1}$
}

Ciliates are unicellular eukaryotes known for their cellular complexity and wide range of natural habitats. How they adapt to their niches and what roles they play in ecology remain largely unknown. The genus Tetrahymena is among the best-studied groups of ciliates and one particular species, Tetrahymena thermophila, is a well-known laboratory model organism in cell and molecular biology, making it an excellent candidate for study in protist ecology. Here, based on cytochrome coxidase subunit I (COX1) gene barcoding, we identify a total of 19 different putative Tetrahymena species and two closely related Glaucoma lineages isolated from distinct natural habitats, of which 13 are new species. These latter include 11 Tetrahymena species found in the bladder traps of Utricularia plants, the most species-rich and widely distributed aquatic carnivorous plant, thus revealing a previously unknown but significant symbiosis of Tetrahymena species living among the microbial community of Utricularia bladder traps. Additional species were collected using an artificial trap method we have developed. We show that diverse Tetrahymena species may live even within the same habitat and that their populations are highly dynamic, suggesting that the diversity and biomass of species worldwide is far greater than currently appreciated.

Ciliated protozoans are unicellular eukaryotic organisms long known for their cellular and behavioral complexity ${ }^{1}$. Several genera (e.g., Tetrahymena, Paramecium, Oxytricha, Stylonychia Euplotes, Blepharisma, and Stentor) have become model taxa for research in many areas of basic biology. Tetrahymena is one such genus and T. thermophila is one of the best-known laboratory eukaryotic models for genetics. It adapts well to growth in axenic proteose-peptone culture and can be easily induced to carry out synchronized sexual conjugation ${ }^{2,3}$. Research driven by the unique biological characteristics of $T$. thermophila has contributed to several scientific milestones including the discovery of catalytic RNA (ribozymes) and telomeres and telomerase ${ }^{4,5}$. Recent annotation of its complete genome will facilitate development of even more tools for genomics research ${ }^{6}$.

Tetrahymena is typically found in freshwater habitats such as ponds, lakes, rivers, and streams ${ }^{7-10}$. Like most protists, closely related Tetrahymena species lacked morphological distinctions and have been difficult to analyze in early studies. DNA barcoding of mitochondrial cytochrome c oxidase subunit I (COX1) has become a powerful molecular tool for identifying species of Tetrahymena ${ }^{11,12}$. The latest study using this tool identified more than 30 new Tetrahymena species collected from sites in the U.S.A ${ }^{13}$, and now a total of 81 named species are listed in the NCBI's GenBank database.

Most known Tetrahymena species have been collected from open freshwater habitats and are considered free-living bacterivores. A number of species have been described as being closely associated with a variety of metazoan (primarily invertebrate) hosts such as snails, slugs, mussels, culicine mosquitoes, chironomid larvae, as well as inhabiting the wounds of amphibians or fishes, ${ }^{7,14}$. The exact relationships between these Tetrahymena species and their metazoan hosts have not yet been well described. In addition, the various morphological forms and complex lifecycles of Tetrahymena indicate that they possess diverse biological characteristics allowing them to cope with many environmental challenges. For example, some Tetrahymena can undergo encystment to survive harsh environments or change their oral morphology (from microstome bacterivore to macrostome carnivore) in

${ }^{1}$ Institute of Molecular Biology, Academia Sinica, Taipei, Taiwan. ${ }^{2}$ Genomics Research Center, Academia Sinica, Taipei, Taiwan. Correspondence and requests for materials should be addressed to M.-C.Y. (email: mcyao@gate. sinica.edu.tw) 
response to food availability ${ }^{1,7,8}$. Current knowledge of Tetrahymena biology is limited to a few laboratory strains that have been selected for particular traits of interest in scientific research. Topics like how it interacts with metazoan hosts and how it adapts to natural environments requires further study to elucidate. Moreover, even Tetrahymena species with highly-similar morphological forms can be divergent for feeding on different bacteria and adapting to different microbial niches. A recent publication reveals the landscape of rapidly evolving genes in Tetrahymena and suggests that different Tetrahymena species have evolved species-specific genes that may regulate their expression to adapt to environmental changes ${ }^{18}$. Taken together, the basic ecology and natural history of Tetrahymena species remains largely unclear.

Recently, one new species, Tetrahymena utriculariae, was reported as a specialized bacterivore from the bladder traps of the aquatic carnivorous plant Utricularia reflexa ${ }^{19,20}$. The genus Utricularia uses its bladder traps to capture and digest small organisms to obtain nutrients. Interestingly, apart from prey items, many living organisms have been observed in these bladder traps ${ }^{21,22}$. Indeed, Charles Darwin reported "In all cases, the bladders with decayed remains swarmed with living Algae of many kinds, Infusoria, and other low organisms, which evidently lived as intruders" ${ }^{23}$, wherein "Infusoria" represents a collective term for aquatic microorganisms and primarily ciliate protozoans. Recent studies indicate that a diverse microbial community exists within Utricularia bladder traps, with cohabiting ciliates perhaps representing commensals feeding on this community or on decaying remains ${ }^{24-26}$. However, the abundance and diversity of ciliates living in Utricularia bladder traps remains to be fully established.

In this partial survey, we report a total of 19 different putative Tetrahymena species and two closely related Glaucoma lineages from Taiwan, 13 of which are new species. These Tetrahymena species were recovered from bladder traps of seven different species of Utricularia plants, indicating a previously unknown but significant symbiotic relationship between Utricularia plants and Tetrahymena ciliates in natural environments. We propose that Tetrahymena has adopted a specialized feeding behavior that involved adapting to the commensal microbial community in Utricularia bladder traps, possibly allowing it to play a critical role as a scavenger in this particular environment. This scavenging behavior is evidenced by the capture of large numbers of Tetrahymena using an artificial trapping method. Our survey has also suggested that Tetrahymena species populations from a single pond are dynamic, and that the biodiversity of Tetrahymena species is much greater than currently acknowledged.

\section{Results}

Tetrahymena species inhabit Utricularia bladder traps. Initially, we collected two species of Utricularia ( $U$. aurea and $U$. gibba) from a small abandoned agricultural irrigation pond located in Taipei, Taiwan $\left(25^{\circ} 07^{\prime} 41.1^{\prime \prime} \mathrm{N} 121^{\circ} 38^{\prime} 09.8^{\prime \prime} \mathrm{E}\right)$. We observed ciliate-like microorganisms actively swimming inside the bladder traps of these plants (Supplementary Movie 1). We examined the bladder traps of $U$. aurea and found that about 90\% of them harbored Tetrahymena-like ciliates. Optical microscopy revealed these unknown ciliates as being morphologically similar to Tetrahymena thermophila, exhibiting its characteristic pear-shaped body and swimming by undulating numerous cilia. DAPI staining of the nuclei revealed nuclear dimorphism, with some individuals possessing two types of cell nuclei (a micronucleus and a macronucleus, which is a unique feature of ciliates) and others only having a macronucleus (which may represent amicronucleate Tetrahymena species) ${ }^{27}$.

To better identify the species of ciliate, we employed COX1 barcoding. We adopted the criteria of Tetrahymena species that strains exhibiting $<1 \%$ COX 1 sequence divergence are considered the same species and those with $>4 \%$ sequence divergence to be different species ${ }^{13,27}$. Among the 18 clones analyzed, we obtained 18 COX1 sequences that could be grouped into two clusters that diverged by about $10.5 \%$, suggesting that they represent two different species. We performed a BLAST search against known COX1 sequences in the NCBI GenBank database and the most similar sequence to ours was COX1 accession GU439296 from Tetrahymena thermophila. However, our sequence (BT-4 in Figs 1 and 2) still diverges from that accession by about 9\%, indicating that our sequence is from a species not represented in the database and is potentially a new species. Our other sequence (BT-1 in Figs 1 and 2) had a closest match to COX1 accession EF070291 of Tetrahymena malaccensis, but still exhibited a sequence difference of about $3 \%$, indicating that it may potentially be a new species.

This preliminary assessment prompted us to expand the survey and collect more Utricularia from around Taiwan, the distribution of which has been previously reported ${ }^{28,29}$.

Tetrahymena is abundant in Utricularia bladder traps. Our extended Utricularia survey yielded 19 samples from nine locations in the north, south, and east of Taiwan, and included seven different species of Utricularia (Table 1 and Fig. 3). We collected 130 Tetrahymena-like ciliate clones from 15 of the 19 Utricularia samples. COX1 barcoding revealed that all these isolates belonged to the genus Tetrahymena (Fig. 2), with the 130 isolates yielding 20 different COX1 sequences that could be grouped into 11 different putative species based on phylogenetic analysis (Fig. 1). We have named these putative species BT-1 to 11 to represent that the Tetrahymena species had been collected from the bladder traps of Utricularia plants. Any given species may be represented by multiple COX 1 haplotypes in the population, but most of these haplotypes are likely to differ by less than $1 \%$. However, BT-5 exhibited particularly pronounced haplotype diversity in which the difference was much larger than $1 \%$ for most isolates, thus they could also be considered as potential new species (Fig. 1). We downloaded all available Tetrahymena COX1 sequences which represent the known named species from the GenBank database to generate a phylogenetic tree incorporating all of our new Tetrahymena sequences. This analysis revealed that BT-11 belongs to the "paravorax" clade, whereas all others belong to the "borealis" clade, and none of our sequences could be identified as belonging to the "australis" clade (Fig. 2) 7,13,30,31. This analysis also allowed us to establish that BT-3 is either T. gruchyi or T. farahensis and that BT-5 and BT-6 are T. tropicalis (an ambiguous taxon exhibiting $\sim 6.2 \%$ intraspecific COX1 sequence divergence among many isolates listed in the GenBank database). BT- 1 is potentially a new species closely related to T. malaccensis, from which it has a COX1 sequence divergence of $\sim 3 \%$, and our other seven new sequences likely represent new species. These results indicate that large 


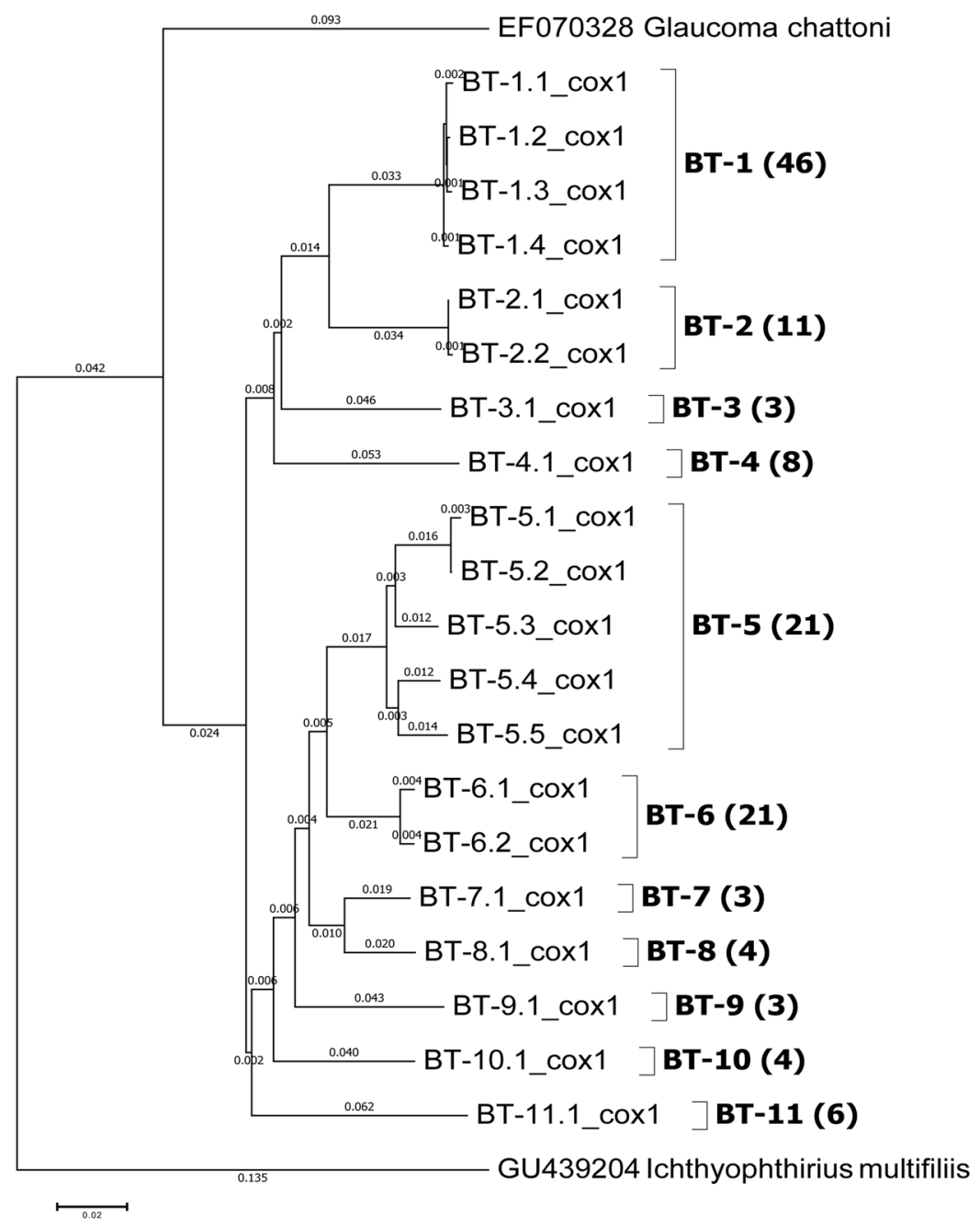

Figure 1. Classification of Tetrahymena ciliates isolated from Utricularia bladder traps. Phylogenetic analysis to classify and group species of Tetrahymena ciliate isolated from Utricularia bladder traps. The analysis involved 22 COX1 sequences, including 20 from samples collected from the wild. Ichthyophthirius multifiliis was used as an outgroup and Glaucoma chattoni was used as a closely-related species. All ambiguous positions were removed from each sequence pair, resulting in a dataset of 1030 nucleotide positions. The Neighbor-Joining tree is drawn to scale, with branch lengths (shown on branches) in the same units as evolutionary distances based on $C O X 1$ sequences. Numbers of Tetrahymena isolates for each lineage are shown in brackets. Scale bar: the number of base substitutions per site.

numbers of diverse and hitherto unknown Tetrahymena species inhabit Utricularia bladder traps and our data confirm the significant relationship between Utricularia plants and Tetrahymena ciliates in natural environments.

Biogeography of Tetrahymena species in Taiwan. Our partial survey provides a preliminary picture of the biogeographic distribution of Tetrahymena species in Taiwan, based on Utricularia sampling sites (Fig. 3). The genus Tetrahymena is globally distributed, but there is strong evidence for population structuring in T. thermophila $^{32,33}$. Among our collection of Tetrahymena specimens, the BT-1, BT-5 and BT-6 lineages are relatively widespread in the northern and southern areas of Taiwan. In contrast, the BT-2, BT-4, BT-9 and BT-10 lineages are restricted to the north of the island, BT-3, BT-7 and BT-11 occur only in the south, and BT- 8 is an eastern lineage.

The symbiotic relationship of Tetrahymena and Utricularia. To better understand the relationship between Tetrahymena and Utricularia, we further assessed the habitats from which our eleven Tetrahymena lineages had been collected (Table 1). Our results show that multiple Tetrahymena species can be found in the same Utricularia species. For example, we identified four different species from U. gibba (BT-1, BT-2, BT-3 and BT-4). Moreover, different species of Utricularia can harbor the same Tetrahymena species. For instance, BT-1 was isolated from both $U$. gibba and $U$. aurea plants. These findings indicate a lack of species specificity in their interrelationship, but we cannot rule out the possibility that certain Tetrahymena species may be better adapted or exhibit a preference for particular species of Utricularia. Thus, the relationship between Tetrahymena and Utricularia may be best described as facultative symbiosis. The highly abundant and diverse Tetrahymena species we found living in Utricularia bladder traps may also imply that they possess unique physiological features that 


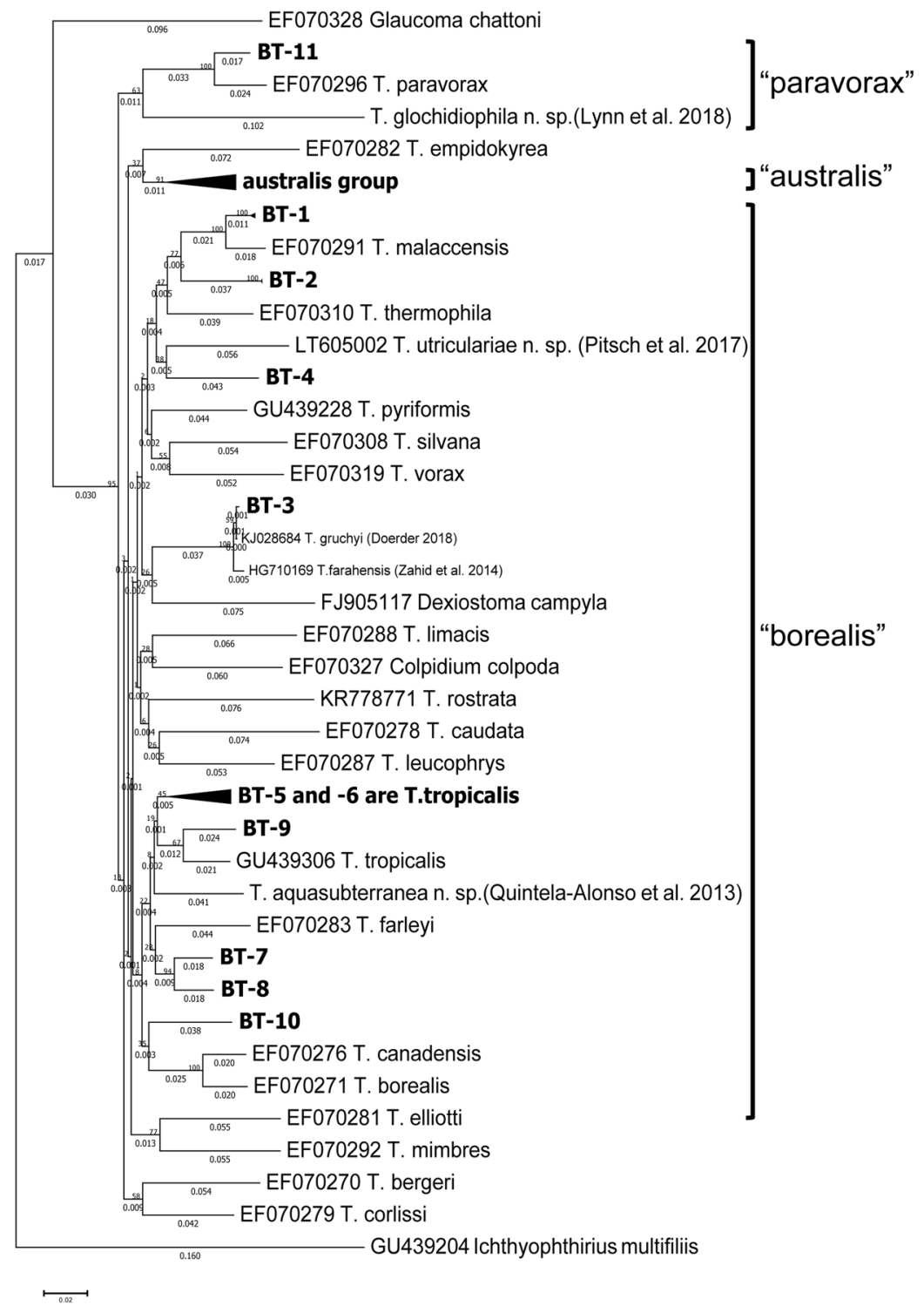

Figure 2. Phylogenetic analysis of Tetrahymena species isolated from bladder traps and named Tetrahymena species. Phylogenetic analysis of 67 Tetrahymena isolates based on COX1 gene sequences, including 20 wild isolates grouped into 11 lineages (BT1 11), 43 named Tetrahymena species, three closely-related species (Glaucoma chattoni, Dexiostoma campyla and Colpidium colpoda), and Ichthyophthirius multifiliis as an outgroup. The COX1 sequences are listed in Supplementary Table 2. The three main clades of Tetrahymena are shown on the right. All ambiguous positions were removed from each sequence pair, resulting in a dataset of 768 nucleotide positions. The Neighbor-Joining tree is drawn to scale, with branch lengths (shown on branches) in the same units as evolutionary distances based on COX1 sequences. Scale bar: the number of base substitutions per site.

\begin{tabular}{|l|l|}
\hline Utricularia species & Tetrahymena species BT \\
\hline U. gibba $(5)$ & $1,2,3,4$ \\
\hline U. striatula (4) & $5,6,8,10$ \\
\hline U. aurea (2) & 1,4 \\
\hline U. bafida (1) & 2,9 \\
\hline U. involvens (1) & $5,6,7,11$ \\
\hline U. calycifida (1) & 6 \\
\hline U. heterosepala $(1)$ & 5,6 \\
\hline
\end{tabular}

Table 1. Tetrahymena species isolated from bladder traps of various Utricularia species. Table showing the species of Utricularia plants collected in this study (number of specimens in brackets) and the corresponding lineages of Tetrahymena isolated from their bladder traps. 


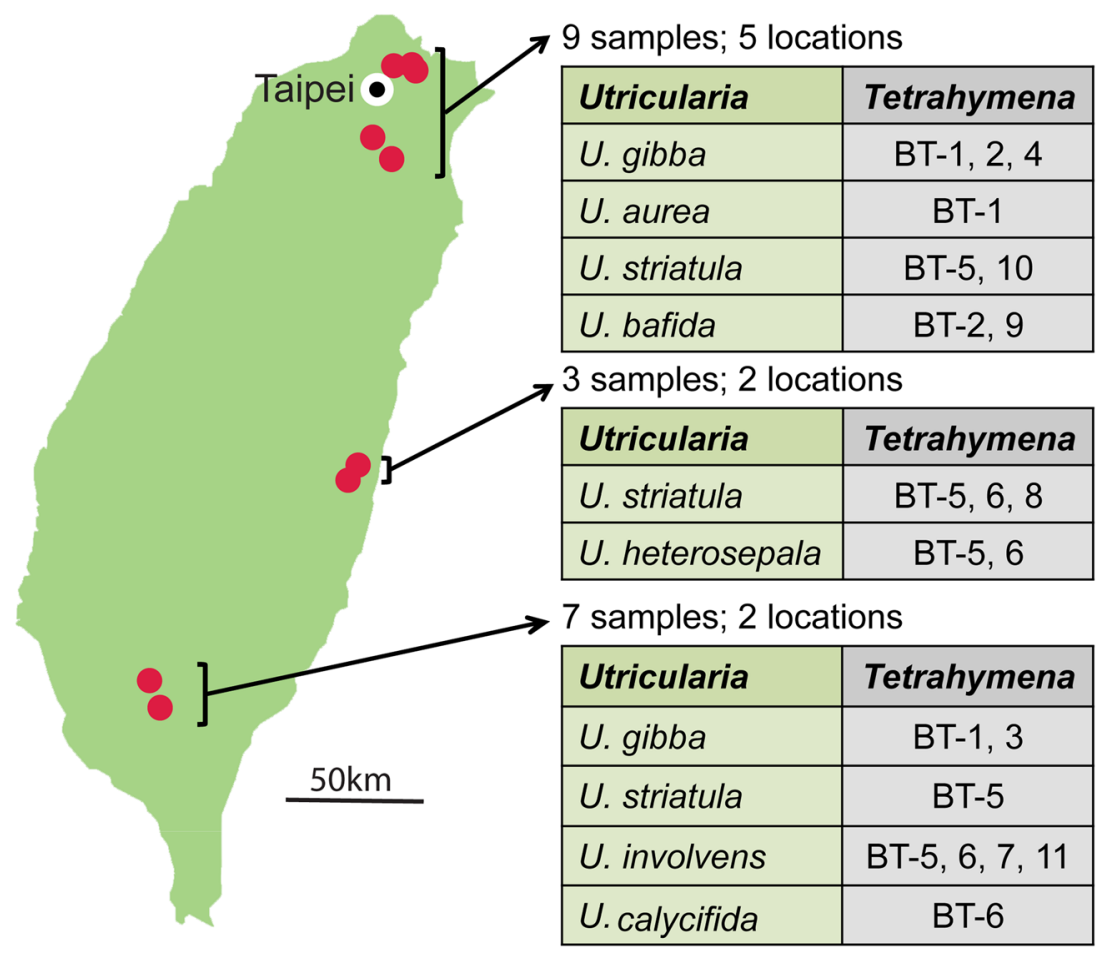

Figure 3. Sampling locations for Utricularia plants in Taiwan. The map of Taiwan shows the sampling locations (red dots) for Utricularia plant samples. Tables at right show which Tetrahymena species were isolated from which species of Utricularia.

facilitates this symbiosis. Moreover, observations of the wild samples suggested that these Tetrahymena may feed on the decaying animal prey in the bladder traps (Supplementary Movies 2 and 3). We also observed that cultured Tetrahymena tended to attach strongly to animal tissue fibers present in the culture medium (Supplementary Movie 4). Indeed wild-caught specimens of Tetrahymena displayed remarkable adaptation to growing in our axenic culture medium that mainly comprised proteose-peptone of animal tissue origin. These observations suggest that Tetrahymena may exhibit scavenging behavior.

To assess this possibility further, we designed a simple "artificial trap" containing a piece of animal tissue as bait to attract Tetrahymena ciliates. We placed our trap in the open water of a pond for $\sim 16$ hours and then examined in the laboratory which organisms had been attracted into the chamber. In total, we examined eight samples ( six from the pond located at $25^{\circ} 02^{\prime} 32.2^{\prime \prime} \mathrm{N} 121^{\circ} 36^{\prime} 46.3^{\prime \prime} \mathrm{E}$; the other two from $25^{\circ} 02^{\prime} 43.0^{\prime \prime} \mathrm{N} 121^{\circ} 36^{\prime} 48.5^{\prime \prime} \mathrm{E}$ and $25^{\circ} 07^{\prime} 41.1^{\prime \prime} \mathrm{N} 121^{\circ} 38^{\prime} 09.8^{\prime \prime} \mathrm{E}$, respectively.), all of which harbored Tetrahymena-like ciliates and yielded 185 isolated clones. Based on our COX1 barcoding protocol, these 185 clones represent 30 different COX 1 haplotypes that could be grouped into 13 different putative species (Supplementary Fig. 1). We have named these putative species AT-1 to 13 to represent that they were collected using our artificial trapping method. Phylogenetic analysis revealed that AT-7 and AT-8 clustered in the "paravorax" clade and that AT-3 and AT-6 grouped in the "australis" clade. The remaining lineages lay in the "borealis" clade, except for two divergent species, AT-12 and AT-13, that grouped with the genus Glaucoma (Supplementary Fig. 2). AT-1 could be identified as either T. gruchyi or T. farahensis. AT- 2 (which is the same as BT- 1 species by the criteria of $<1 \%$ COX 1 sequence divergence) is potentially a new species closely related to T. malaccensis, from which its COX 1 sequence diverged by $\sim 3 \%$. AT- 3 , AT-4 and AT-6 appear to represent T. shanghaiensis, T. pyriformis and T. alphapoecilia SIN, respectively, and the remaining eight lineages may be new species. These results indicate that an abundant and diverse Tetrahymena community exists in the pond, and that artificial trapping is an efficient collection technique, as alluded to in a previous report ${ }^{8}$.

Different natural habitats harbor divergent Tetrahymena communities. When we compared the Tetrahymena species collected from our two natural habitats, symbiotes in bladder traps and artificially trapped free-living ciliates in the open water of a pond, we found that the communities only overlapped marginally. Of the 21 distinct lineages identified using both approaches, only three had been isolated using both methods: BT-1 and AT-2, BT-3 and AT-1, BT- 11 and AT-7 (each pair share a similar COX1 sequence of $<1 \%$ differences) (Fig. 4). This outcome suggests that distinct lineages of Tetrahymena may inhabit various natural environments. This supposition is supported by the fact that we have identified seven different Tetrahymena species from three types of natural habitats in and around one pond in Taipei, Taiwan $\left(25^{\circ} 07^{\prime} 41.1^{\prime \prime} \mathrm{N} 121^{\circ} 38^{\prime} 09.8^{\prime \prime} \mathrm{E}\right)$; two inhabited Utricularia bladder traps, four were found in the open water, and one was isolated from a small pool of water in a tree crevice (Supplementary Fig. 3). 
(a)

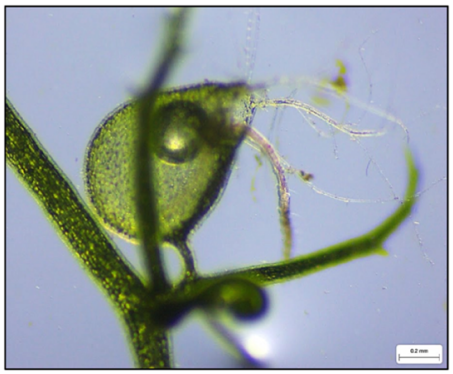

(b)

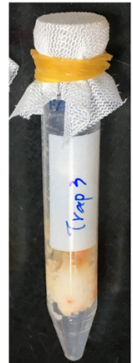

(c)

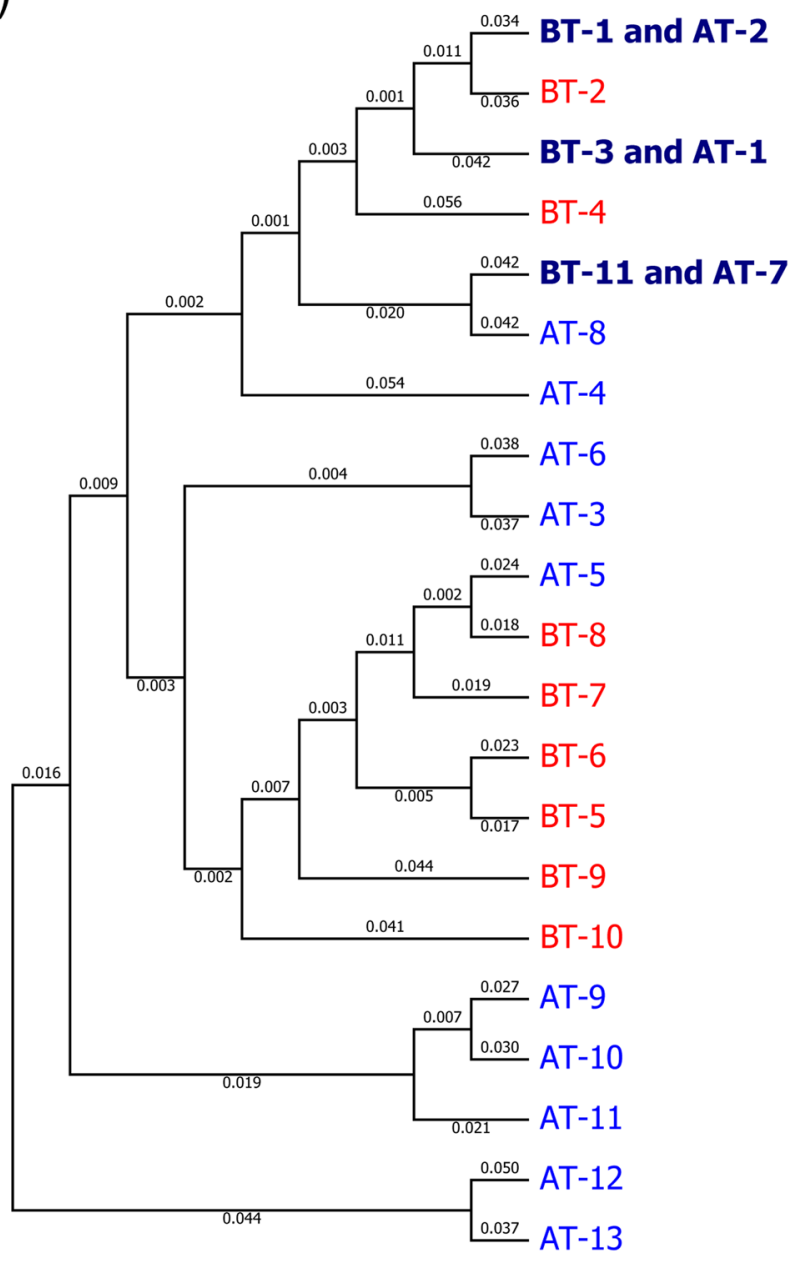

Figure 4. Comparison of Tetrahymena species collected from Utricularia bladder traps and by artificial trapping. (a) The image shows a bladder trap of Utricularia gibba. (b) The image shows an artificial trap. (c) The topology of the phylogenetic tree for Tetrahymena species was generated by the Neighbor-Joining algorithm based on COX1 gene sequences. All ambiguous positions were removed for each sequence pair, resulting in a dataset of 994 nucleotide positions. Red labels show species only isolated from Utricularia bladder traps. Pale blue labels identify species only isolated by the artificial trapping method. Dark blue labels represent overlapping species, i.e., isolated from both collection methods.

Sympatric Tetrahymena species populations dynamically change within a pond. To better understand the dynamics of Tetrahymena populations at a single location, we repeatedly sampled Tetrahymena species using our artificial trapping method at a single pond in the grounds of Academia Sinica, Taipei, Taiwan $\left(25^{\circ} 02^{\prime} 32.2^{\prime \prime} \mathrm{N} 121^{\circ} 36^{\prime} 46.3^{\prime \prime} \mathrm{E}\right)$. We collected six samples over a period of eight months, i.e. in March, April, May, August, September and October of 2018. We recovered four Tetrahymena species (AT-1, AT-3, AT-9 and AT-11) in the March sample, five species (AT-1, AT-3, AT-6, AT-11 and AT-12) in the April sample, three species (AT1, AT-3 and AT-6) in the May sample, one species (AT-1) in the August sample, one species (AT-13) in the September sample, and three species (AT1, AT-3 and AT-5) in the October sample (Fig. 5). This preliminary survey showed that up to six different Tetrahymena species and two different Glaucoma species were found at a 


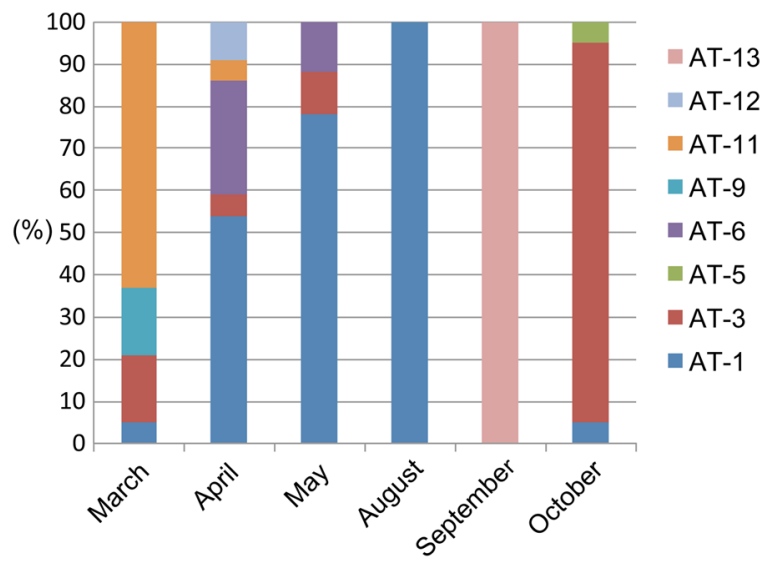

Figure 5. Dynamic changes in a Tetrahymena community sampled from a single location in a pond. Relative abundances (\%, y-axis) of Tetrahymena trapped in different months (March, April, May, August, September and October of 2018) from a single pond in Academic Sinica, Taipei, Taiwan.

single location in the pond, but species combinations varied throughout the year. Together, our findings indicate that the number of Tetrahymena species could be greatly underestimated by single field surveys at a sole location, and imply that global diversity of Tetrahymena species may be much greater than currently acknowledged.

\section{Discussion}

In this study, we investigated pond habitat and Utricularia plant bladder traps and found a diversity of Tetrahymena ciliates. We consider the interrelationship between Tetrahymena and Utricularia to be a facultative symbiosis. Tetrahymena appears to be a commensal organism in the microbial community of Utricularia bladder traps. It can directly feed on axenic culture medium made from animal tissues, and an artificial trap with decaying animal tissue bait attracted Tetrahymena ciliates. Thus, we conclude that Tetrahymena species exhibit strong scavenging behavior, indicating that the ecological role of Tetrahymena might be as "a scavenger", assisting in the process of animal tissue decomposition in the food web and facilitating energy recycling in the ecosystem. It would be interesting to investigate which physiological features contribute to Tetrahymena's scavenging behavior. For instance, Tetrahymena possesses a secretory organelle called the "mucocyst" that harbors proteases, and it has been linked to responses to changing environments ${ }^{34,35}$.

Aside from North America, previous surveys of Tetrahymena species have been rather limited. Some species have been collected from parts of Asia, but none from Taiwan. This study represents the first on the genus Tetrahymena in Taiwan, a subtropical East Asian island. We report 19 Tetrahymena species and two closely related Glaucoma lineages, 13 of which may be new species. This diversity in natural environments suggests that the island may harbor a rich biodiversity for this protist. Broader surveys will be necessary to establish the biodiversity of Taiwanese Tetrahymena, and expanding the survey to other islands in the region will likely prove fruitful.

In this study, we found that only two among the 19 Tetrahymena species identified belonged to the "australis" clade, which was considered to be less diversified with closely related species than the "borealis" clade ${ }^{7,13}$. This could represent an interesting biogeographical or habitant specialization. However, it is also possible that our collection methods are biased toward one clade. These issues can be addressed by altering collecting methods and performing a wider range of field survey.

Little is known about the dynamics of Tetrahymena ciliate populations in nature and what impacts they have on ecosystems. In this study, we identified a diverse Tetrahymena community inhabiting a single location in a pond, indicating that large numbers of sympatric species can live together. This result also indicates that Tetrahymena populations may actively compete for resources in the wild. Further investigations will be necessary to assess the interplay among different Tetrahymena populations and reveal their ecological roles in the wild. Moreover, we found Tetrahymena communities to be dynamic, perhaps changing in response to the environment. It would be very informative to develop long-term monitoring to track ciliate communities over time and reveal which factors (such as location, weather, season or human activity) impact Tetrahymena population dynamics in nature.

\section{Methods}

Utricularia plant collection and Tetrahymena-like ciliate examination. Utricularia plant samples were collected from different locations in Taiwan that are listed in Supplementary Table 1. A simple examination was conducted to verify whether Tetrahymena-like ciliates exist in the bladder traps of Utricularia plants. We dissected and isolated individual bladder traps from the stem of Utricularia plants and placed them separately in the wells of 96- well plate containing standard proteose-peptone culture medium for T. thermophila. The existing Tetrahymena-like ciliates would be cultivated in the well if they were released from the bladder traps. The medium drop plate can replace the 96-well plate for better observations and manipulations under a dissecting microscope.

Tetrahymena ciliate isolation. Based on the standard protocol for inbreeding T. thermophila strains used in the laboratory, wild collecting ciliates were cultivated by standard SPP or Neff medium and kept to grow at 
room temperature in a moist incubator. We conducted single-cell isolation from the cultured Tetrahymena-like ciliates, which were isolated from Utricularia bladder traps, artificial trapping in the pond, and various habitat samples in which the microbial communities could contain multiple species. Isolated single-cell was cultivated in a small drop $(\sim 20-30 \mu \mathrm{l})$ of Neff medium for several days. The pure Tetrahymena clones were established and generated when they adapted to Neff medium. Each established pure clone was prepared to extract genomic DNA when the cultured cell density reached $\sim 2 \times 10^{5}$ cells $/ \mathrm{ml}$.

The artificial trapping method. A simple "artificial trap" is designed as a chamber (a $15 \mathrm{ml}$ plastic FALCON tube) containing a piece of animal meat (we used a piece of shrimp meat about 0.5 cubic centimeters in size for all sample collections) as bait to attract Tetrahymena ciliates. The opening to the chamber was covered with a filter (Nylon cloth, the hole size is about $0.5 \mathrm{~mm}$ ) to prevent larger carnivores from entering it. We placed the "artificial trap" device in shallow water areas, approximately $10-20 \mathrm{~cm}$ deep at the bottom of the pond for $\sim 16$ hours. After retrieving to the laboratory, immediately the fluid of the trapping chamber was poured into cell culture plates and the antibiotics and antifungals were added to prevent the over-growth of bacteria and fungi. The cultured Tetrahymena-like ciliates usually became readily detectable in 1-3 days.

COX1 barcoding and phylogenetic relationship analysis. Tetrahymena genomic DNA were extracted by a QIAGEN commercial kit and used as templates to perform polymerase chain reactions (PCR) to amplify the partial COX1 gene using the primer set, the forward primer COI-FW 5'-ATGTGAGTTGATTTTATAGAGCAGA $-3^{\prime 12}$ and the reverse primer FolB $5^{\prime}$-TAAACTTCAGGGTGACCAAAAAATCA- $3^{\prime 36}$. The PCR products were purified and sequenced to obtain about $1 \mathrm{~kb}$ length of a partial COX 1 sequence. The phylogenetic analysis was conducted by using the Neighbor-Joining method ${ }^{37}$. The percentage of replicate trees in which the associated taxa clustered together in the bootstrap test (1000 replicates) are shown next to the branches ${ }^{38}$. The tree was drawn to scale, with branch lengths (next to the branches) in the same units as those of the evolutionary distances used to infer the phylogenetic tree. The evolutionary distances were computed using the Kimura 2-parameter method ${ }^{39}$ and were in the units of the number of base substitutions per site. All ambiguous positions were removed for each sequence pair. Evolutionary analyses were conducted in MEGA $7^{40}$.

\section{References}

1. Lynn, D. H. The Ciliated Protozoa: Characterization, Classification, and Guide to the Literature. 3rd Edition edn, (Springer, 2008).

2. Ruehle, M. D., Orias, E. \& Pearson, C. G. Tetrahymena as a Unicellular Model Eukaryote: Genetic and Genomic Tools. Genetics 203, 649-665, https://doi.org/10.1534/genetics.114.169748 (2016).

3. Cassidy-Hanley, D. M. Tetrahymena in the Laboratory: Strain Resources, Methods for Culture, Maintenance, and Storage. Method Cell Biol 109, 239-276, https://doi.org/10.1016/B978-0-12-385967-9.00008-6 (2012).

4. Greider, C. W. \& Blackburn, E. H. Identification of a specific telomere terminal transferase activity in Tetrahymena extracts. Cell 43, 405-413 (1985)

5. Kruger, K. et al. Self-splicing RNA: autoexcision and autocyclization of the ribosomal RNA intervening sequence of Tetrahymena. Cell 31, 147-157 (1982).

6. Eisen, J. A. et al. Macronuclear genome sequence of the ciliate Tetrahymena thermophila, a model eukaryote. PLoS biology 4, e286, https://doi.org/10.1371/journal.pbio.0040286 (2006).

7. Lynn, D. H. \& Doerdert, F. P. The Life and Times of Tetrahymena. Method Cell Biol 109, 11-27, https://doi.org/10.1016/B978-0-12385967-9.00002-5 (2012).

8. Doerder, F. P. \& Brunk, C. Natural populations and inbred strains of Tetrahymena. Methods Cell Biol 109, 277-300, https://doi. org/10.1016/B978-0-12-385967-9.00009-8 (2012).

9. Simon, E. M., Nanney, D. L. \& Doerder, F. P. The “Tetrahymena pyriformis" complex of cryptic species. Biodivers Conserv 17, 365-380, https://doi.org/10.1007/s10531-007-9255-6 (2008).

10. Elliott, A. M. Distribution of Tetrahymena-Pyriformis. J Protozool 17, 162-\&, https://doi.org/10.1111/j.1550-7408.1970.tb02348.x (1970).

11. Kher, C. P. et al. Barcoding Tetrahymena: discriminating species and identifying unknowns using the cytochrome coxidase subunit I (cox-1) barcode. Protist 162, 2-13, https://doi.org/10.1016/j.protis.2010.03.004 (2011).

12. Chantangsi, C. et al. Barcoding ciliates: a comprehensive study of 75 isolates of the genus Tetrahymena. Int J Syst Evol Micr 57, 2412-2425, https://doi.org/10.1099/ijs.0.64865-0 (2007).

13. Doerder, F. P. Barcodes Reveal 48 New Species of Tetrahymena, Dexiostoma, and Glaucoma: Phylogeny, Ecology, and Biogeography of New and Established Species. The Journal of eukaryotic microbiology. https://doi.org/10.1111/jeu.12642 (2018).

14. Struder-Kypke, M. C., Wright, A. D. G., Jerome, C. A. \& Lynn, D. H. Parallel evolution of histophagy in ciliates of the genus Tetrahymena. Bmc Evolutionary Biology 1, Artn 5, https://doi.org/10.1186/1471-2148-1-5 (2001).

15. Batson, B. S. A Paradigm for the Study of Insect Ciliate Relationships - Tetrahymena-Sialidos Sp-Nov (Hymenostomatida, Tetrahymenidae), Parasite of Larval Sialis-Lutaria (Linn) (Megaloptera, Sialidae). Philos T Roy Soc B 310, 123-\&, https://doi. org/10.1098/rstb.1985.0102 (1985).

16. Batson, B. S. Tetrahymena-Dimorpha Sp-Nov (Hymenostomatida, Tetrahymenidae), a New Ciliate Parasite of Simuliidae (Diptera) with Potential as a Model for the Study of Ciliate Morphogenesis. Philos T Roy Soc B 301, 345-\&, https://doi.org/10.1098/ rstb.1983.0027 (1983)

17. Corliss, J. O. Natural infection of tropical mosquitoes by ciliated protozoa of the genus Tetrahymena. Trans R Soc Trop Med Hyg $\mathbf{5 5}$, 149-152 (1961)

18. Xiong, J. et al. Hidden genomic evolution in a morphospecies-The landscape of rapidly evolving genes in Tetrahymena. PLoS biology 17, doi:ARTN e3000294, https://doi.org/10.1371/journal.pbio.3000294 (2019).

19. Simek, K. et al. Ecological Traits of the Algae-Bearing Tetrahymena utriculariae (Ciliophora) from Traps of the Aquatic Carnivorous Plant Utricularia reflexa. The Journal of eukaryotic microbiology 64, 336-348, https://doi.org/10.1111/jeu.12368 (2017).

20. Pitsch, G. et al. The Green Tetrahymena utriculariae n. sp. (Ciliophora, Oligohymenophorea) with Its Endosymbiotic Algae (Micractinium sp.), Living in Traps of a Carnivorous Aquatic Plant. The Journal of eukaryotic microbiology 64, 322-335, https://doi. org/10.1111/jeu.12369 (2017).

21. Adamec, L. The smallest but fastest: ecophysiological characteristics of traps of aquatic carnivorous Utricularia. Plant Signal Behav 6, 640-646 (2011).

22. Sirova, D. et al. Microbial community development in the traps of aquatic Utricularia species. Aquat Bot 90, 129-136, https://doi. org/10.1016/j.aquabot.2008.07.007 (2009).

23. Darwin, C. R. Insectivorous Plants., (John Murray, London, 1875). 
24. Alcaraz, L. D., Martinez-Sanchez, S., Torres, I., Ibarra-Laclette, E. \& Herrera-Estrella, L. The Metagenome of Utricularia gibba’s Traps: Into the Microbial Input to a Carnivorous Plant. PloS one 11, doi:ARTN e0148979, https://doi.org/10.1371/journal. pone.0148979 (2016).

25. Plachno, B. J., Lukaszek, M., Wolowski, K., Adamec, L. \& Stolarczyk, P. Aging of Utricularia traps and variability of microorganisms associated with that microhabitat. Aquat Bot 97, 44-48, https://doi.org/10.1016/j.aquabot.2011.11.003 (2012).

26. Sirova, D. et al. Correction to: Hunters or farmers? Microbiome characteristics help elucidate the diet composition in an aquatic carnivorous plant. Microbiome 7, 4, https://doi.org/10.1186/s40168-018-0617-y (2019).

27. Doerder, F. P. Abandoning sex: multiple origins of asexuality in the ciliate Tetrahymena. BMC evolutionary biology 14, 112, https:// doi.org/10.1186/1471-2148-14-112 (2014).

28. Hsu, T. C., Chen, Z. H. \& Chaos, Y. S. New Additions of the Bladderworts (Lentibulariaceae) in Taiwan. Taiwania 62, 99-104, https://doi.org/10.6165/tai.2017.62.99 (2017).

29. Yang, Y. P., Liu, H. Y. \& Chao, Y. S. Trap gland morphology and its systematic implications in Taiwan Utricularia (Lentibulariaceae). Flora 204, 692-699, https://doi.org/10.1016/j.flora.2008.09.009 (2009).

30. Chantangsi, C. \& Lynn, D. H. Phylogenetic relationships within the genus Tetrahymena inferred from the cytochrome coxidase subunit 1 and the small subunit ribosomal RNA genes. Molecular Phylogenetics and Evolution 49, 979-987, https://doi.org/10.1016/j. ympev.2008.09.017 (2008).

31. Nanney, D. L., Park, C., Preparata, R. \& Simon, E. M. Comparison of sequence differences in a variable 23S rRNA domain among sets of cryptic species of ciliated protozoa. Journal of Eukaryotic Microbiology 45, 91-100, https://doi.org/10.1111/j.1550-7408.1998. tb05075.x (1998).

32. Zufall, R. A., Dimond, K. L. \& Doerder, F. P. Restricted distribution and limited gene flow in the model ciliate Tetrahymena thermophila. Mol Ecol 22, 1081-1091, https://doi.org/10.1111/mec.12066 (2013).

33. Foissner, W., Chao, A. \& Katz, L. A. Diversity and geographic distribution of ciliates (Protista: Ciliophora). Biodivers Conserv 17, 345-363, https://doi.org/10.1007/s10531-007-9254-7 (2008).

34. Guerrier, S., Plattner, H., Richardson, E., Dacks, J. B. \& Turkewitz, A. P. An evolutionary balance: conservation vs innovation in ciliate membrane trafficking. Traffic 18, 18-28, https://doi.org/10.1111/tra.12450 (2017).

35. Turkewitz, A. P. Out with a bang! Tetrahymena as a model system to study secretory granule biogenesis. Traffic 5, 63-68, https://doi. org/10.1046/j.1600-0854.2003.00155.x (2004).

36. Folmer, O., Black, M., Hoeh, W., Lutz, R. \& Vrijenhoek, R. DNA primers for amplification of mitochondrial cytochrome c oxidase subunit I from diverse metazoan invertebrates. Mol Mar Biol Biotechnol 3, 294-299 (1994).

37. Saitou, N. \& Nei, M. The neighbor-joining method: a new method for reconstructing phylogenetic trees. Mol Biol Evol 4, 406-425, https://doi.org/10.1093/oxfordjournals.molbev.a040454 (1987).

38. Felsenstein, J. Confidence Limits on Phylogenies: An Approach Using the Bootstrap. Evolution 39, 783-791, https://doi. org/10.1111/j.1558-5646.1985.tb00420.x (1985).

39. Kimura, M. A simple method for estimating evolutionary rates of base substitutions through comparative studies of nucleotide sequences. J Mol Evol 16, 111-120 (1980).

40. Kumar, S., Stecher, G. \& Tamura, K. MEGA7: Molecular Evolutionary Genetics Analysis Version 7.0 for Bigger Datasets. Mol Biol Evol 33, 1870-1874, https://doi.org/10.1093/molbev/msw054 (2016).

\section{Acknowledgements}

We thank members of the Yao lab for helpful discussions. We thank other members of the community for their assistance to collect Utricularia plant samples, particularly Mr. Chien-Jung Lin, Assistant Researcher in Taiwan Forestry Research Institute, Fushan Research Center in northern Taiwan and Mr. Jui-Hsuan Kuo, Senior Collection Manager and Dr. Chia-Wei Li, CEO of Dr. Ceclila Koo Botanic Conservation center in southern Taiwan. We received funding from the Institute of Molecular Biology, Academia Sinica of Taiwan (to M.C.Y.).

\section{Author Contributions}

C.Y.C., S.L.C., I.T.L. and M.C.Y. designed and performed research; C.Y.C. collected and analyzed data; C.Y.C. and M.C.Y. wrote the paper.

Additional Information

Supplementary information accompanies this paper at https://doi.org/10.1038/s41598-019-50123-1.

Competing Interests: The authors declare no competing interests.

Publisher's note Springer Nature remains neutral with regard to jurisdictional claims in published maps and institutional affiliations.

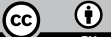

Open Access This article is licensed under a Creative Commons Attribution 4.0 International License, which permits use, sharing, adaptation, distribution and reproduction in any medium or format, as long as you give appropriate credit to the original author(s) and the source, provide a link to the Creative Commons license, and indicate if changes were made. The images or other third party material in this article are included in the article's Creative Commons license, unless indicated otherwise in a credit line to the material. If material is not included in the article's Creative Commons license and your intended use is not permitted by statutory regulation or exceeds the permitted use, you will need to obtain permission directly from the copyright holder. To view a copy of this license, visit http://creativecommons.org/licenses/by/4.0/.

(C) The Author(s) 2019 